\title{
A novel micro-channel cooler for high-power diode laser arrays
}

\author{
A. Kozłowska, ${ }^{11}$ M. Teodorczyk, ${ }^{1}$ E. Dąbrowska-Tumańska, ${ }_{1}^{1}$ Marcin Chmielewski, ${ }^{1}$ Dariusz Podniesiński, ${ }^{1}$ \\ and A. Maląg ${ }^{1}$ \\ ${ }^{1}$ Institute of Electronic Materials Technology, Optoelectronics Department, Wólczyńska 133, 01-919 Warszawa,
}

Received August 19, 2014; accepted March 26, 2014; published March 31, 2014

\begin{abstract}
The construction of a novel micro-channel cooler that allows for high packaging density of laser diode arrays is presented. Its main element is the micro-channel copper plate with micro-pipes that transport cooling liquid in the vicinity of a diode laser array. A prototype series of the coolers was manufactured and tested. Experimental arrangements for the investigation of thermal properties and micro-photoluminescence measurements of the devices were built. Experimental results indicate that the proposed cooler is an interesting solution for high-power diode laser arrays.
\end{abstract}

High-power diode laser arrays (DLA) are efficient devices that find broad applications in pumping for solid state lasers, medicine, direct material processing including cutting, welding and heat treating. The electrical-tooptical efficiencies of the devices emitting at $0.8-1.0 \mu \mathrm{m}$ are typically in the range of $50-75 \%$. Efficient cooling is critical for proper operation of these devices because of high power densities that are encountered. Their operation temperatures need to be kept at a moderate level of 40$60^{\circ} \mathrm{C}$ in order to avoid problems with accelerated degradation. This implies strict requirements for the cooling of the devices.

Diode laser arrays are typically mounted on stackable micro-channel [1-2] or impingement-type heat exchangers [3]. In commercial systems dominate copper microchannel heat sinks with deionized water as the cooling medium. The channel diameters are usually $200-500 \mu \mathrm{m}$ of width, and the distance between the laser diode array and the water channel is $100-300 \mu \mathrm{m}$. During recent years research has concentrated on construction optimizations in order to increase the reliability of the devices and insure more efficient cooling, including ceramic, erosion resistant approaches [4] and various design modifications [5-6].

In order to increase the total output optical power, the arrays are often combined into the stacks. A high packaging density diode laser stack operating under continuous-wave condition at an output power of $1200 \mathrm{~W}$ with a bar pitch of $1.8 \mathrm{~mm}$ was demonstrated [7]. The distance between the arrays in the stack ought to be as small as possible and miniaturized constructions are in demand. From the point of view of long-term reliability of

\footnotetext{
*E-mail: anna.kozlowska@itme.edu.pl
}

diode laser arrays the minimization of packaging induced strain is of paramount importance. The strain arises during soldering the diode laser array due to the mismatch of the coefficient of thermal expansion (CTE) between the heat sink and the DLA material. The conventional CTE matched solutions use $\mathrm{CuW}$ and AlN submounts, in more advanced systems multilayer $\mathrm{Cu} / \mathrm{Mo} / \mathrm{Cu}$ or $\mathrm{CuC}$ submounts are applied [8-9].

In this Letter, a novel construction of micro-channel cooler for high-power diode laser arrays is presented. In comparison to conventional micro-channel coolers, the novel cooler is composed of fewer material layers, due to a modified water circulation path through the inserted micro-pipes, which makes the minimization of the cooler thickness possible. The distance between the cooling area and the laser diode array is here restricted only by the thickness of the submount placed directly above the water channels. The concept, manufacturing method as well as the experimental results are presented.

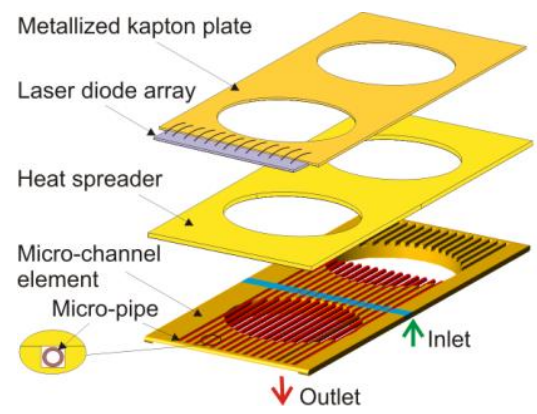

b)

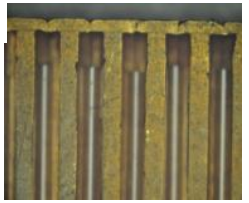

Fig. 1. (a) The schematic of a miniaturized micro-channel cooler, (b) photograph of a microchannel element with glass micropipes.

The scheme of the micro-channel cooler is shown in Fig. 1a. Micro-channels are produced in a copper plate and in each channel a micro-pipe is placed. The inlet and outlet parts are separated in the middle of the cooler. The cooler is closed with a heat spreading plate that is made from copper or from the material with the coefficient of thermal expansion matched to the diode laser array material. On 
the top of the assembly, the diode laser array is soldered and the electrical connections are provided. The cooling fluid flows in the micro-pipes to the region close to the laser diode array. Next, it is transported to the outlet port through the areas between the walls of rectangular channels and the outer pipe surface. The module construction of the cooler allows to form a diode laser array stack.

According to the scheme shown in Fig. 1a a prototype series of micro-channel coolers was manufactured. First, the rectangular channels were performed in copper plates using a numerically controlled saw. The ends of the channels were closed with electrolytic grade copper using a specially elaborated method and the corrosion resistant layers were deposited. In each channel one borosilicate glass micro-pipe was inserted (Fig. 1b). The choice of the corrosion resistant micro-pipe material is beneficial from the point of view of the reliability of the coolers. The $200 \mu \mathrm{m}$ thick heat spreaders were bonded to the microchannel elements using heat curing epoxy adhesive. The dimension of the cooling structure in the direction perpendicular to the junction of the laser diode array is $1 \mathrm{~mm}$. A reference copper plate or a metal matrix composite foil $\mathrm{Al}_{2} \mathrm{O}_{3}-\mathrm{Cu}$ was used as a heat spreader . The geometrical details of the cooler considered in this work are given in Table 1.

On each cooler a laser diode array with dimensions $10 \mathrm{~mm} \times 1 \mathrm{~mm} \times 0.1 \mathrm{~mm}$ was soldered epi-side down, using indium. Electrical wires were bonded by ultracompression to the metal coated Kapton elements. The results for three exemplary devices labelled L1, L2 and L3 are presented in this Letter. Device L1 comprises a commercial laser diode array built from 66 individual emitters, soldered on a copper plate. Devices L2 and L3 are based on a Double Barrier Separate Confinement Single Quantum Well structure [10] and contain 16 single emitters each. Device $\mathrm{L} 2$ uses a $20 \% \quad \mathrm{Al}_{2} \mathrm{O}_{3}-\mathrm{Cu}$ composite heat spreader, whereas L3 is soldered on the reference copper plate.

Table 1.
\begin{tabular}{|c|c|c|c|c|c|}
\hline $\begin{array}{c}\text { Width of } \\
\text { channel } \\
(\mathrm{mm})\end{array}$ & $\begin{array}{c}\text { Depth of } \\
\text { channel } \\
(\mathrm{mm})\end{array}$ & $\begin{array}{c}\text { Width of } \\
\text { fin }(\mathrm{mm})\end{array}$ & $\begin{array}{c}\text { Number } \\
\text { of channels }\end{array}$ & $\begin{array}{c}\text { Outer } \\
\text { diameter of } \\
\text { micro-pipe } \\
(\mathrm{mm})\end{array}$ & $\begin{array}{c}\text { Inner } \\
\text { diameter of } \\
\text { micro-pipe } \\
(\mathrm{mm})\end{array}$ \\
\hline 0.37 & 0.41 & 0.31 & 14 & 0.32 & 0.20 \\
\hline
\end{tabular}

The experimental arrangement for thermal characterisation of the cooling system is presented in Fig. 2a. As a cooling medium, deionized water is used. The gear pump MCP-Z with the pump head Z-1830 (Ismatec) is used to circulate the working fluid. A filter is used to remove unwanted particles. The micro-channel cooler is placed on the test assembly containing a water cooled heat exchanger and electrical connections for the laser diode array. Inlet water temperature is stabilized using a TEC cooler. The inlet and outlet fluid temperatures are monitored using thermocouples. The

http://www.photonics.pl/PLP temperature of the diode laser array is determined using the THERMOSENSORIK 640 SM camera equipped with a $640 \times 512$ pixel InSb-detector (wavelength range 1.1$5.3 \mu \mathrm{m})$. The IR-lens with a focal length $\mathrm{f}=28 \mathrm{~mm}$ is used. The calibration characteristics are determined for each characterized DLA. For this purpose a non-operating diode laser array is heated externally by TEC and chiller (both of them operating in the heating mode). For each temperature setting a thermal image of the device is captured with a camera. The calibration data are approximated by a third-order polynomial.

(a)

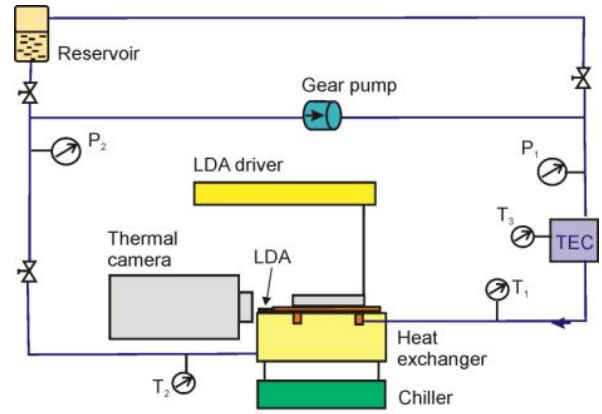

(b)

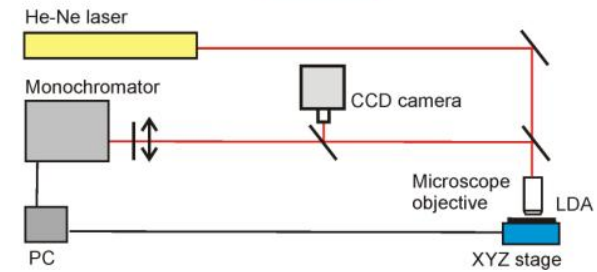

Fig. 2. An experimental arrangement for the thermal characterization of the cooling system (a) and for micro-photoluminescence measurements (b).

Figure $2 b$ presents the micro-photoluminescence ( $\mu$-PL) set-up used in this work. As an excitation a helium-neon laser emitting at $632.8 \mathrm{~nm}$ is employed. Its beam is focused on the active region of the laser diode array using a microscope objective with the magnification $\times 20$. The laser beam spot on the DLA mirror is observed by a CCD camera. The photoluminescence signal is analysed by a monochromator with a photomultiplier using a phase sensitive detection scheme. The DLA is placed on the DC motor controlled translation stage.

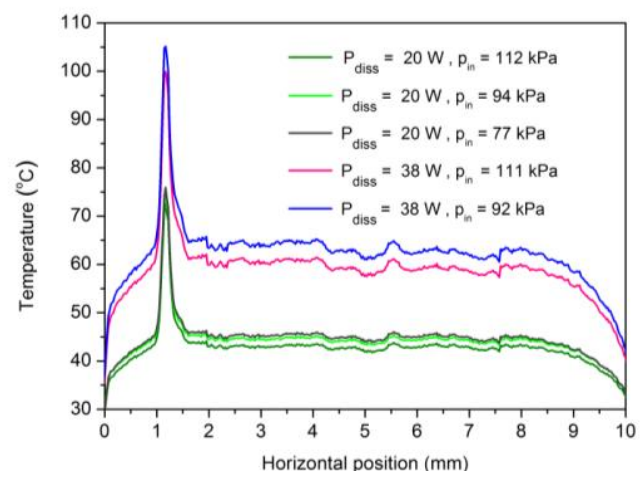

Fig. 3. In-junction plane temperature profiles for the device L1 for different flow rates of cooling medium (and different input pressures) and two dissipated power levels of the diode laser array.

(C) 2014 Photonics Society of Poland 
Using the experimental arrangement shown in Fig. 2a temperature maps were recorded for various operating conditions, e.g. for several flow rates of the cooling medium and different power levels of the laser diode array. Figure 3 presents the results of the thermographic characterization of device L1. An increase in the flow rate (and increased input pressure) resulted in a decrease in the operational temperature.

In the middle part of the array, uniform temperature profiles (with the exception of a hot spot in the left part of the array) were obtained. The dissipated thermal power was $20 \mathrm{~W}$ and $38 \mathrm{~W}$. The inspection of the laser front facet under an optical microscope revealed a spot at the position corresponding to the observed temperature peak. Most probably, a contaminant absorbing optical radiation acted as a starting point for the extended defect area, leading to the degradation of the emitter.

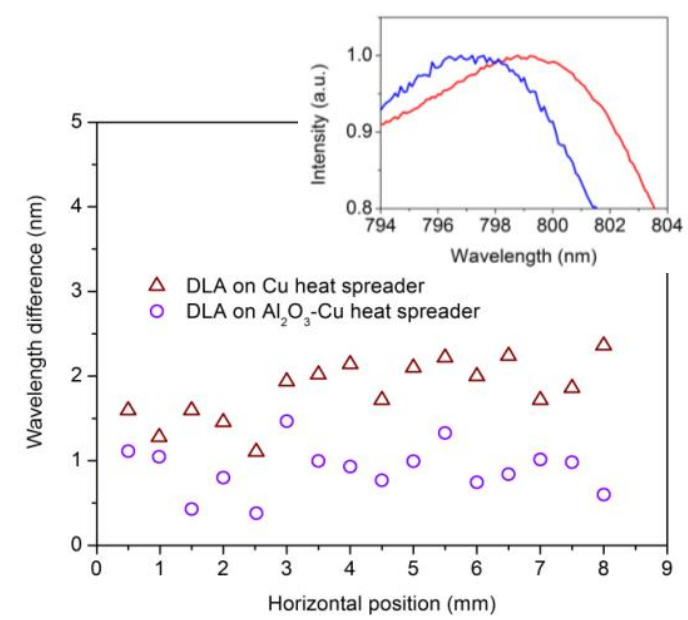

Fig. 4. PL maximum wavelength difference of unmounted and packaged DLAs. Inset shows the part of PL spectrum for an exemplary emitter of umounted (red line) and packaged (blue line) device L3.

Figure 4 shows the results of micro-photoluminesce experiments for devices L2 and L3. For each emitter in the DLA under test, the $\mu$-PL spectra of the QW emission are measured twice - first, for an unmounted device and then, after soldering the array on the cooler. Due to packaging induced strain the blue shift of the spectrum is observed. The inset in Fig. 4 illustrates this effect showing the central part of the PL spectrum for the exemplary emitter of the L3 device before (red line) and after (blue line) soldering the array. The resulting spectrum maximum wavelength difference is plotted for each emitter of L2 and L3 laser diode arrays.

The blue shift PL maximum of $0.07 \mathrm{~nm}$ is responsible for the compressive strain of $1 \mathrm{MPa}$ (for bulk GaAs) [8]. Thus, the results indicate that in both cases we observe packaging induced strain, although indium as a "soft" solder compensates this effect significantly and typically for the copper heat sinks, the "smile effect" is not observed. The use of a metal matrix composite $\mathrm{Al}_{2} \mathrm{O}_{3}-$ $\mathrm{Cu}$ heat spreader allowed to lower the packaging induced strain significantly. However, the observed spread of the data indicates the inhomogeneity of the epitaxial layers of DLA.

Further improvements of the proposed prototype microchannel cooler will concentrate on lowering the thermal resistance of the package, e.g. by replacing epoxy bonding by much more advantageous, from the thermal point of view, soldering or direct bonding of cooler elements. The optimization of expansion matched metal matrix composite heat-spreaders is underway.

In summary, we have proposed novel the construction of a micro-channel cooler for high power diode laser arrays. The small dimensions of the cooler in the direction perpendicular to the junction allows forming an array stack with an array-to array distance (pitch) of $1 \mathrm{~mm}$. This is advantageous for optical pumping applications and superior in comparison to convectional constructions, where the pitch of the array stack ranges typically from $1.6 \mathrm{~mm}$ to $1.9 \mathrm{~mm}$ [11-13].

The results of thermal characterization and microphotoluminescence measurements have been shown. The presented experimental results indicate that the cooling system is an interesting solution for high-power diode laser arrays due to its miniaturized construction and lower induced strain in comparison to conventional copper coolers.

This work was supported by National Centre for Research and Development under the research project N R02 0004 06. Marta Doch is gratefully acknowledged for her contribution in the experimental part of the work.

\section{References}

[1] V.K. Krause et al., Proc. SPIE 2148, 351 (1994).

[2] Y. Karni, G. Klumel, M. Levy, Y. Berk, Y. Openhaim, Y. Gridish et al., Proc. SPIE 6876, 687604-1 (2008).

[3] J. Vetrovec, Proc. SPIE 6876, 687603-1 (2008).

[4] R. Feeler, Proc SPIE 6876, 687608-1-8 (2008).

[5] J. Dix, A. Jokar, R. Martinsen, Proc. SPIE 6876, 687606-1-10 (2008).

[6] J. Wang et al., Proc. SPIE 8241, 82410H-1-9 (2012).

[7] Z. Liu, G. Fang, K. Feng, Chin. Opt. Lett. 7, 214 (2009).

[8] J.W. Tomm, J. Jimenez, Eds, Quantum-well laser array packaging (New York, McGraw-Hill 2007).

[9] M. Leers, K. Boucke, Proc. IEEE Electr. Comp. Techn. Conf. 1011 (2008).

[10] A. Maląg, A. Jasik, M. Teodorczyk, A. Jagoda, A. Kozłowska, IEEE Photon. Tech. Lett. 18, 1582 (2006).

[11] http://www.coherent.com

[12] http://www.jenoptik.com

[13] http://www.dilas.com 